\title{
VARIATION IN THE EPIPROCT OF ARSAPNIA DECEPTA BANKS, 1897 (PLECOPTERA: CAPNIIDAE), WITH COMMENTS ON ARSAPNIA COYOTE (NELSON \& BAUMANN 1987)
}

\author{
Richard W. Baumann ${ }^{1} \&$ Bill P. Stark ${ }^{2}$ \\ ${ }^{1}$ Department of Biology and Monte L. Bean Life Science Museum, \\ Brigham Young University, Provo, Utah, U.S.A. 84602 \\ E-mail: richard_baumann@byu.edu \\ ${ }^{2}$ Department of Biology, Box 4045, Mississippi College, Clinton, Mississippi, U.S.A. 39058 \\ E-mail: stark@mc.edu
}

\begin{abstract}
Epiprocts and tergal processes were examined using scanning electron microscopy for scattered populations of Arsapnia decepta Banks, 1897 and A. coyote (Nelson \& Baumann, 1987) from throughout their known ranges. Epiproct lengths ranged from 379-578 $\mu \mathrm{m}$ among A. decepta males from 19 sites, and from 575-618 $\mu \mathrm{m}$ for A. coyote males from two sites. Among A. decepta males, the numbers of thick spine-like epiproct setae ranged from 10-34 per cluster. We conclude that A. decepta, as currently defined, shows considerable variation in epiproct dimensions, numbers of epiproct setae per cluster, and width of the dorsal process on tergum 7. In addition, the limited data from our small sample of $A$. coyote specimens, and the molecular data published recently by colleagues, support continued recognition of this closely related species. SEM images are presented to document the observed variation in these structures from various populations in western North America.
\end{abstract}

Keywords: Plecoptera, Capniidae, Arsapnia decepta, A. coyote, epiproct morphology, scanning electron microscopy

\section{INTRODUCTION}

The genus Arsapnia was originally proposed by Banks (1897), but was placed as a synonym of Capnia Pictet, 1841 by Claassen (1924), where it remained until Murányi et al. (2014) re-established the group as a valid Nearctic genus based on the eight members of the Arsapnia decepta species group (Nelson \& Baumann 1989). Arsapnia decepta Banks,
1897 is presently known from scattered populations in Arizona, Colorado, Mexico (Baja California; Chihuahua), New Mexico, and Nevada (DeWalt et al. 2017, Jewett 1966, Kondratieff \& Baumann 2002, Nelson \& Baumann 1989, Sargent et al. 1991). Our preliminary observations of epiproct morphology for Eagle Creek, New Mexico and Redstone, Colorado populations showed significant variation 
Baumann, R.W., and B.P. Stark 2017. Variation in the Epiproct of Arsapnia decepta Banks, 1897 (Plecoptera: Capniidae), with Comments on Arsapnia coyote (Nelson \& Baumann 1987). Illiesia, 13(01):1-21. https://doi.org/10.25031/2017/13.01

in numbers of spine-like setae clustered dorsally on the epiproct surface (10-15 vs 19-24 respectively) and epiproct length $(413 \mu \mathrm{m}$ vs $525 \mu \mathrm{m}$ respectively). Consequently, we chose to evaluate epiproct variation with SEM among populations identified as A. decepta from throughout the range of the species. We also comment on A. coyote (Nelson \& Baumann, 1987b), considered the sister species of A. decepta, and known only from southern California where A. decepta has not been reported (Nelson \& Baumann 1987b, 1989).

\section{MATERIALS AND METHODS}

Samples of $A$. decepta stored in $75-80 \%$ ethanol were obtained from the Bill P. Stark collection at Mississippi College, Clinton, Mississippi (BPSC), C.P. Gillette Museum of Arthropod Diversity, Colorado State University, Fort Collins, Colorado (CSUC), and the Monte L. Bean Life Science Museum, Brigham Young University, Provo, Utah (BYUC). Samples from which scanning electron micrographs were made are listed below as "Material examined". No attempt was made to list all localities where $A$. decepta populations are known.

Wings were removed from specimens and the bodies sonicated for 10-15 seconds to remove debris. Cleaned specimens were inspected under a SZH10 Olympus dissecting microscope, or a Wild M-8 stereoscope, and then dehydrated through 90, 95 and $100 \%$ ethanol for 10 minutes each. Subsequently, specimens were transferred to hexamethyldisilizane (HMDS) for 30-60 minutes before mounting on aluminum stubs using double sided adhesive copper tape. Stubs were sputter coated with gold palladium and examined with an Amray 1810 scanning electron microscope at Mississippi College, or a Philips XL30 ESEM FEG microscope at the Brigham Young University Electron Microscopy Laboratory. Epiproct terminology follows that of Nelson \& Baumann (1989).

\section{RESULTS}

\section{Arsapnia decepta Banks, 1897 \\ Shortbeak Snowfly}

\begin{abstract}
Arsapnia decepta Banks, 1897:22. Type series (4 specimens), Fort Collins [Larimer Co.], Colorado (Museum of Comparative Zoology)

Capnia decepta: Claassen, 1924:43.

http://lsid.speciesfile.org/urn:lsid:Plecoptera.speciesfile.org: $\underline{\text { TaxonName: } 4975}$
\end{abstract}

Capnia barbata Frison, 1944:153. Holotype ơ (Illinois Natural History Survey), Longmont [Larimer Co.], Little Thompson River, Colorado (Synonymy Nelson \& Baumann, 1989)

http://lsid.speciesfile.org/urn:lsid:Plecoptera.speciesfile.org: TaxonName:4976

\section{Colorado Populations}

(Figs.1-12)

Material examined. USA: Colorado: Boulder Co., Gregory Creek, Gregory Canyon Trail, 39.9977, 105.2997, 2 February 2015, C. Verdone, $3 \hat{\jmath}$ (CSUC). Larimer Co., Buckhorn Creek, $5 \mathrm{mi}$ above Masonville, 40.5723, -105.4504, 16 March 1991, B.C. Kondratieff, 46ㅊ, 9 ㅇ (CSUC). Cedar Creek, Cedar Creek Road, Forest Road 128, 5.1 miles northeast of Drake, 40.6603, -105.2902, 12 April 2014, B.C. Kondratieff, T.P. Belcher, 100+ $\hat{\sigma}, 100+q$ (BYUC). Redstone Canyon, 40.5870, -105.2659, 24 January 1986, B.C. Kondratieff, $12 \widehat{\jmath}, 18$ (CSUC). Tributary Spring Creek, Fort Collins, 40.5645, -105.1587, 15 February 1986, B.C. Kondratieff, $12 \hat{\jmath}, 3 q$ (CSUC).

Male epiproct $(n=8)$. Length $468-528 \mu \mathrm{m}$, width at midlength 236-303 $\mu \mathrm{m}$; greatest width slightly posterior to midlength. Body of epiproct bearing a pair of expansive median bulbs, forming convex ear-like lobes on either side of median groove near midlength (Figs. 1-2, 7, 11). Width across neck 91$111 \mu \mathrm{m}$. Narrow median groove extends from posterior margin of posterior declivity to epiproct apex, becoming wider near apex (Figs. 2-5, 7, 9, 11). On either side of groove a cluster (12-24) of thick, spine-like setae occurs near origin of anterior declivity (Figs.1-5, 7-9, 11-12). Ventral epiproct 
Baumann, R.W., and B.P. Stark 2017. Variation in the Epiproct of Arsapnia decepta Banks, 1897 (Plecoptera: Capniidae), with Comments on Arsapnia coyote (Nelson \& Baumann 1987). Illiesia, 13(01):1-21. https://doi.org/10.25031/2017/13.01
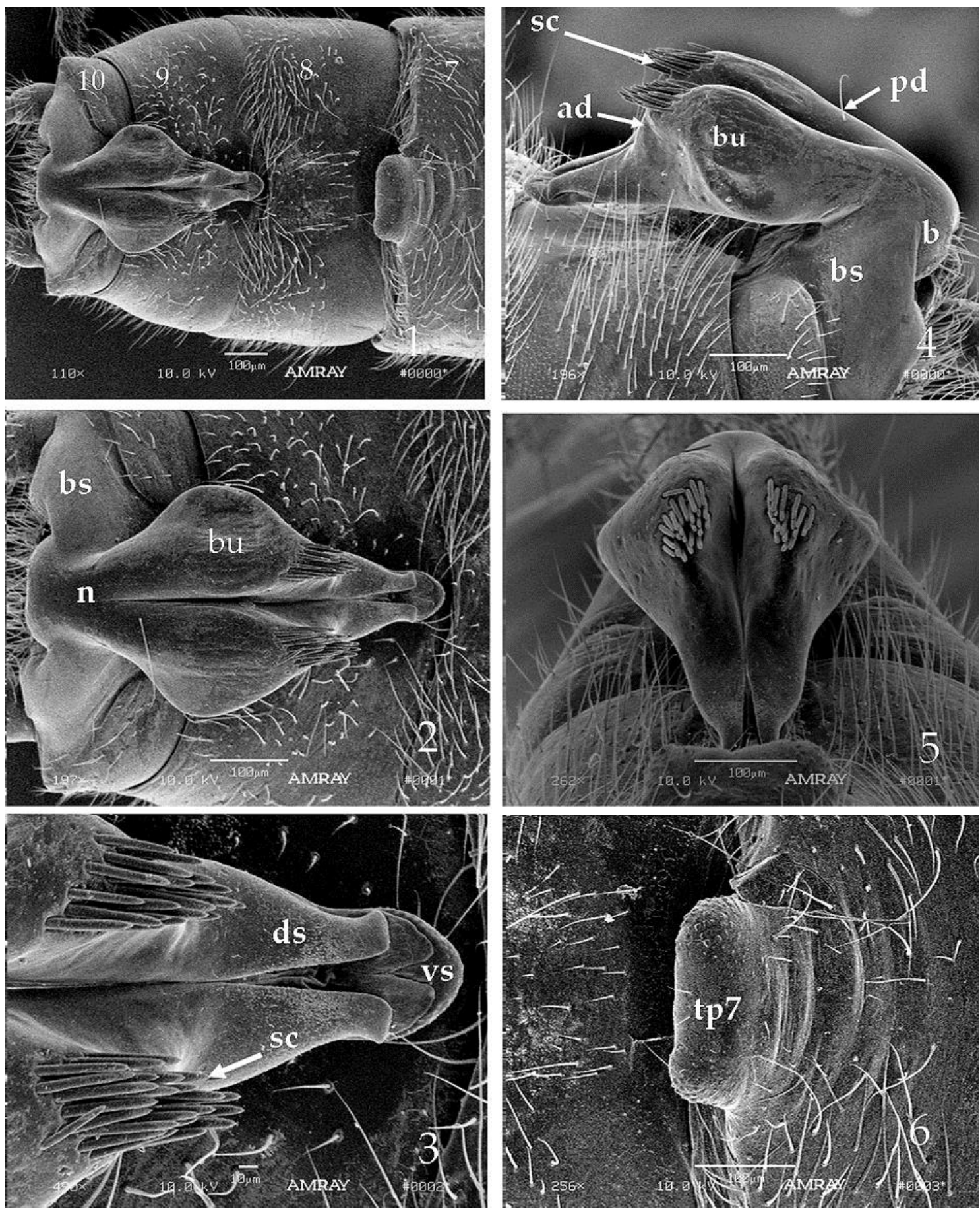

Figs. 1-6. Arsapnia decepta male reproductive structures, Redstone Creek, Larimer Co., Colorado. 1. Abdominal terga 7-10. 2. Epiproct, dorsal. 3. Epiproct apex, dorsal. 4. Epiproct, lateral. 5. Epiproct, anterodorsal. $6.7^{\text {th }}$ tergal process, anterodorsal. $(\mathrm{ad}=$ anterior declivity; $\mathrm{b}=$ base; $\mathrm{bs}=$ basal strut; $\mathrm{bu}=\mathrm{bulb}$; $\mathrm{ds}=$ dorsal sclerite; $\mathrm{n}=$ neck; $\mathrm{pd}=$ posterior declivity; $\mathrm{sc}=$ setal spine cluster; $\mathrm{tp} 7=$ tergal process of segment $7 ; \mathrm{vs}=$ ventral sclerite) . 

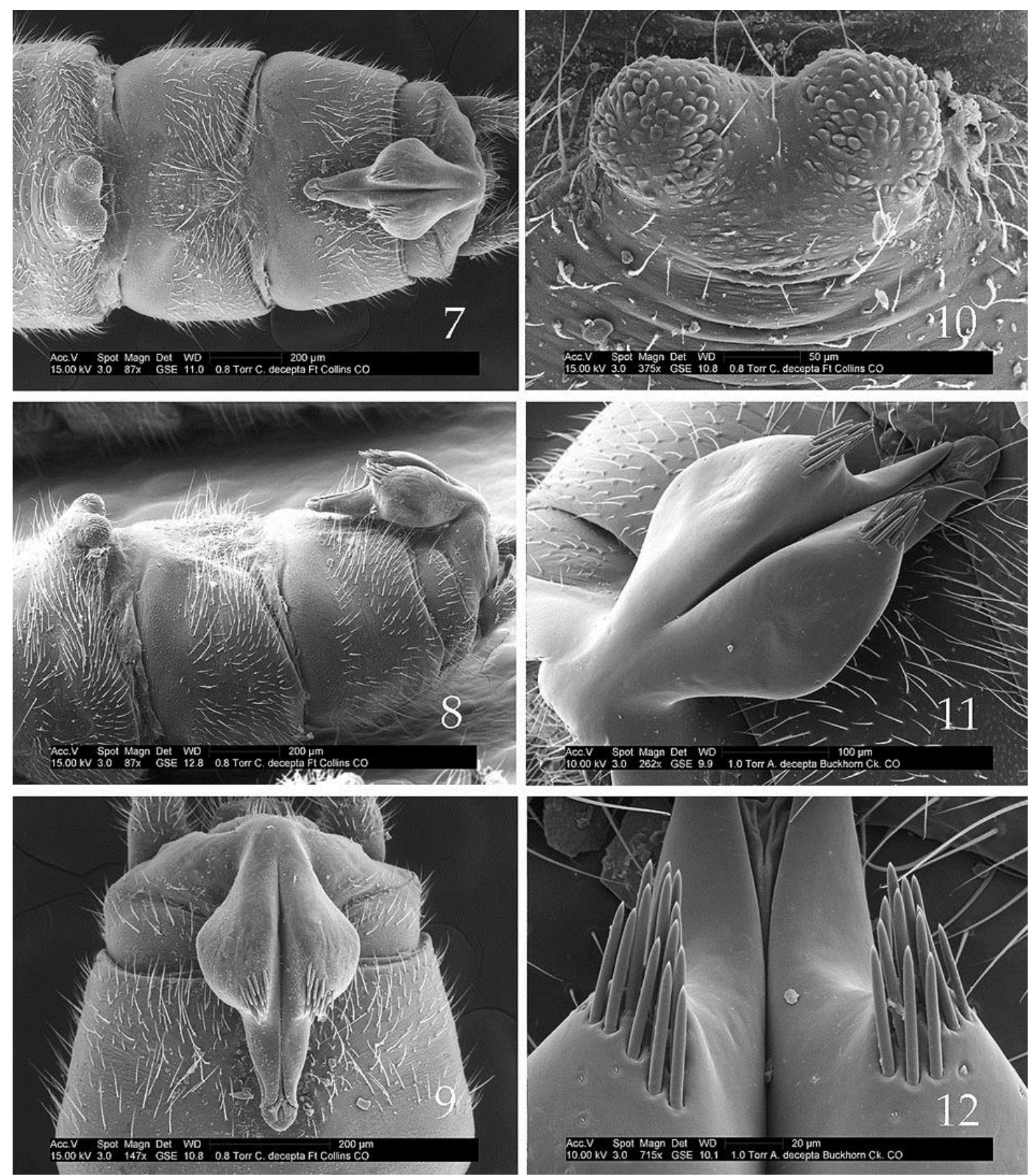

Figs. 7-12. Arsapnia decepta male reproductive structures, 7-10. tributary of Spring Creek, Larimer Co., Colorado, 11-12. Buckhorn Creek, Larimer Co., Colorado. 7. Abdominal terga 7-10. 8. Abdominal segments 7-10, lateral. 9. Male, terminalia, dorsal. 10. $7^{\text {th }}$ tergal process, anterodorsal. 11. Epiproct, dorsal. 12. Epiproct setal clusters. 
sclerite extends $40 \mu \mathrm{m}$ beyond tips of outwardly hooked apices of dorsal sclerite (Figs. 3, 11). Lateral margins of epiproct slightly sinuate between bases of spine-like setae and apex (Figs. 2, 5, 9, 11); lateral aspect of epiproct (Figs. 4, 8) shaped somewhat like a duck head.

Tergal process. A single, mound-shaped process on tergum 7 with dorsal margin entire (Figs. 1, 6), or bearing a shallow dorsal concavity (Fig.10); lateral margins converging only slightly from anterior aspect; process bears lateral patches of small scalelike structures. Width of process across anterior margin 172-231 $\mu \mathrm{m}$; width greater than median height.

Comments. The type locality of $A$. decepta, given as "Ft. Collins, Col." by Banks (1897), remains an unspecified site. The specimens listed from "Redstone Canyon" in Larimer Co. were taken from a stream that forms part of the Big Thompson River drainage perhaps $5-7 \mathrm{~km}$ southwest of the Fort Collins city limits and the specimens listed from "tributary Spring Creek" were collected in the city limits of Fort Collins from a tributary which enters the Cache la Poudre River; we regard the latter group of specimens as topotypes for this species. Colleagues at Colorado State University have records of several hundred specimens collected from sites in Boulder, Douglas, Fremont, Jefferson, Larimer and Pueblo counties (pers. comm. B.C. Kondratieff), and Stark et al. (1973) reported the species (as Capnia barbata) from Littleton in Arapahoe Co. and from the Little Thompson River in Boulder Co. Most Colorado records that we have seen are from sites along the Front Range, but the next nearest sites where populations are known to occur are in the Sangre de Cristo Range of southern Colorado and northern New Mexico (B.C. Kondratieff, pers. comm.; Jacobi \& Baumann 1983). The nearest sites to the Front Range where our specimens were collected are Eagle Creek and Rio Bonito in Lincoln Co., New Mexico.

The epiproct of specimens from Gregory Creek, Boulder Co. have the greatest width (300-303 $\mu \mathrm{m})$ of any specimens examined in the study and the length of all but two of the Front Range specimens exceeds the mean length for the entire sample $($ mean $=487.9 \mu \mathrm{m})($ Tables 1-5, Figs. 1-3). The general shape of the apical third of the epiproct is intermediate between that of Rio Bonito specimens and those of Eagle Creek (Figs. 3, 51-54), however, they are more similar to the Eagle Creek specimens (Fig. 51) that share the longer, more exposed apex.

Table 1. Epiproct and $7^{\text {th }}$ tergal process characteristics for Colorado specimens of Arsapnia decepta.

\begin{tabular}{|l|l|l|l|l|l|l|l|}
\hline \multicolumn{1}{|c|}{ County } & \multicolumn{1}{|c|}{ Site } & $\begin{array}{l}\text { Epiproct } \\
\text { Length } \mu \mathrm{m}\end{array}$ & $\begin{array}{l}\text { Epiproct } \\
\text { Width } \mu \mathrm{m}\end{array}$ & $\begin{array}{l}\text { \# Left } \\
\text { Setae }\end{array}$ & $\begin{array}{l}\text { \# Right } \\
\text { Setae }\end{array}$ & $\begin{array}{l}\text { T7 } \\
\text { Process } \mu \mathrm{m}\end{array}$ & $\begin{array}{l}\text { Length/ } \\
\text { Width Ratio }\end{array}$ \\
\hline Boulder & $\begin{array}{l}\text { Gregory } \\
\text { Creek }(\mathrm{n}=2)\end{array}$ & $524-527$ & $300-303$ & $17-18$ & $17-20$ & 188 & $1.74-1.75$ \\
\hline Larimer & $\begin{array}{l}\text { Buckhorn } \\
\text { Creek }(\mathrm{n}=1)\end{array}$ & 477 & 236 & 14 & 12 & 200 & 2.02 \\
\hline Larimer & $\begin{array}{l}\text { Cedar } \\
\text { Creek }(\mathrm{n}=2)\end{array}$ & 468 & 257 & 14 & 14 & 172 & 1.82 \\
\hline Larimer & $\begin{array}{l}\text { Redstone } \\
\text { Canyon }(\mathrm{n}=2)\end{array}$ & 525 & 285 & 19 & 24 & 198 & 1.86 \\
\hline Larimer & $\begin{array}{l}\text { Tributary } \\
\text { SpringCreek }(\mathrm{n}=1)\end{array}$ & 528 & 272 & 16 & 14 & 231 & 1.94 \\
\hline
\end{tabular}



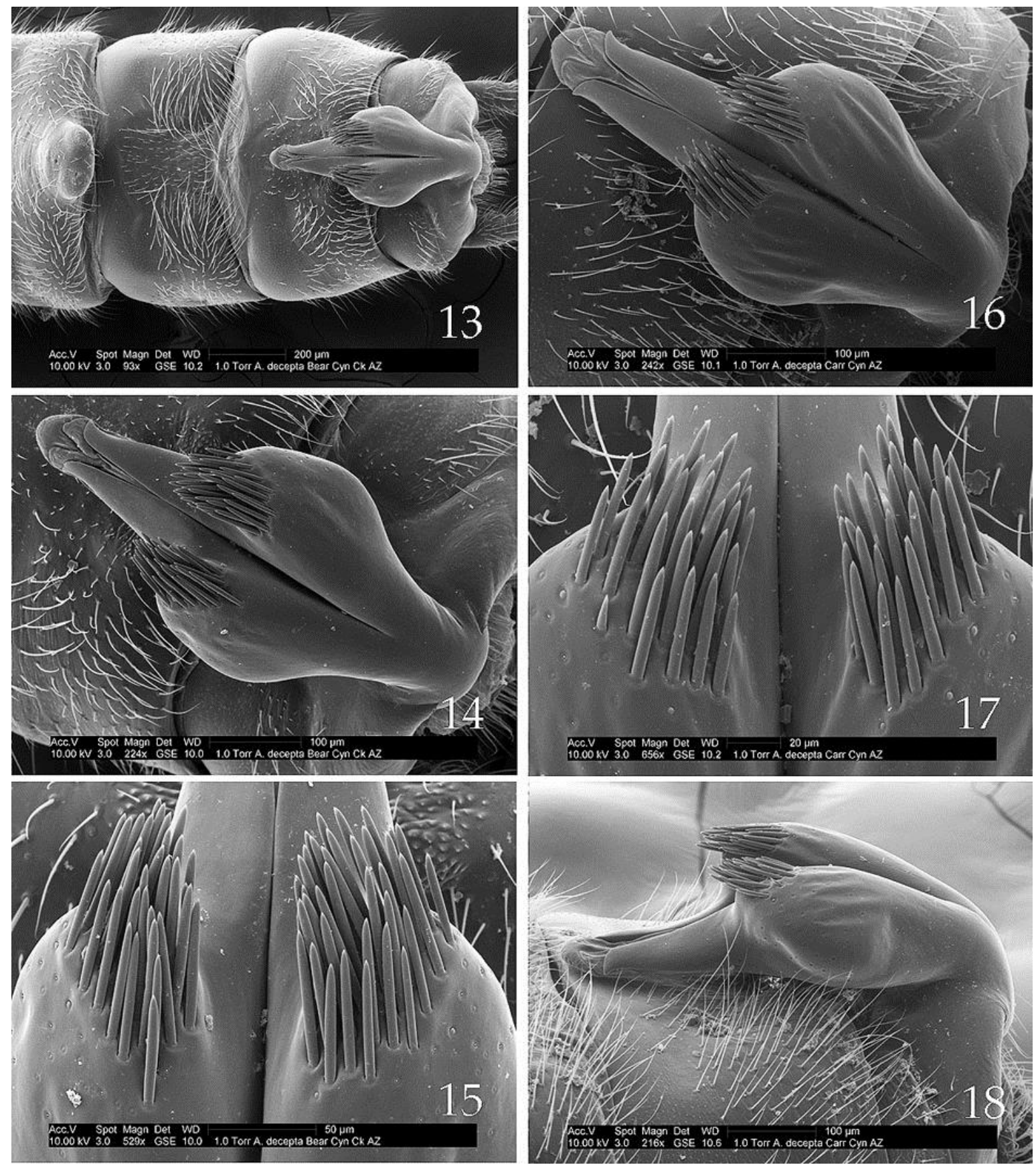

Figs. 13-18. Arsapnia decepta male reproductive structures, 13-15. Bear Canyon, Pima Co., Arizona, 16-18. Carr Canyon, Cochise Co., Arizona.13. Abdominal terga 7-10. 14. Epiproct, dorsal. 15. Setal spine clusters, dorsal. 16. Epiproct, dorsal. 17. Setal spine clusters, dorsal. 18. Epiproct, lateral. 

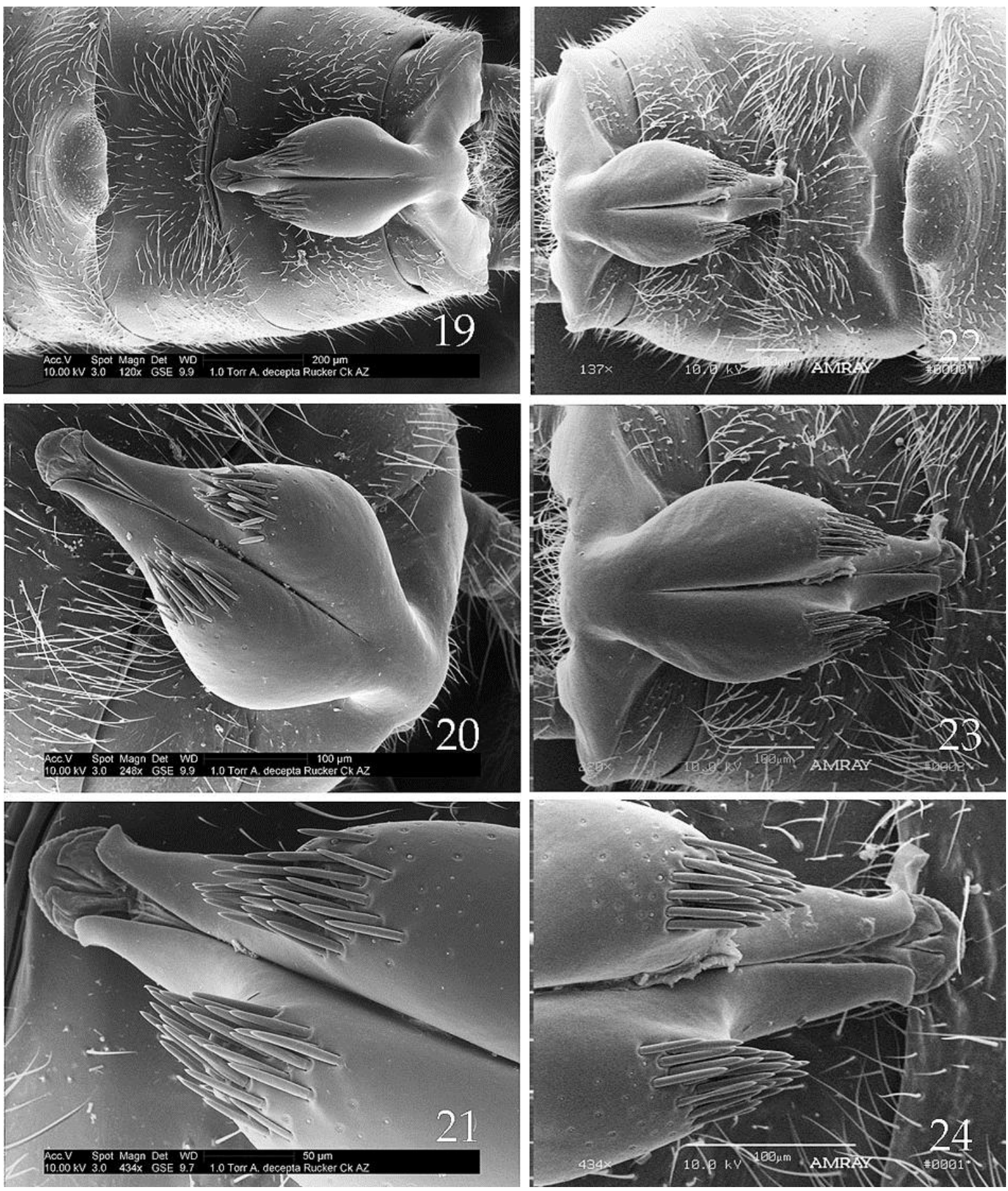

Figs. 19-24. Arsapnia decepta male reproductive structures, 19-21. Rucker Creek, Chiricahua Mountains, Cochise Co., Arizona, 22-24. Cave Creek, Chiricahua Mountains, Cochise Co., Arizona. 19. Abdominal terga 7-10. 20. Epiproct, dorsal. 21. Epiproct apex and setal spine clusters, dorsal. 22. Abdominal terga 7-10. 23. Epiproct dorsal. 24. Epiproct apex and setal spine cluster. 
Arizona Populations

(Figs. 13-45)

Material examined. USA: Arizona: Cochise Co., Cave Creek, Southwest Research Station, Chiricahua Mountains, 5400', 31.8854, -109.2073, 3 January 1972, V. Roth, 3ㅊ, 1우 (BYUC). Rucker Creek, Camp Rucker Campground, Chiricahua Mountains, 31.7615, -109.3695, 17 January 1984, R.W. Baumann, C.R. Nelson, 60, 2 + (BYUC). Carr Creek, Carr Canyon, Huachuca Mountains, 34.4476, -110.2821, 17 January 1984, R.W. Baumann, C.R. Nelson, $7 \AA$, 5 q (BYUC). Coconino Co., Oak Creek, Hwy 89A, above junction of West Fork Oak Creek, 35.0012, -111.7377, 20 January 1988, R.W. Baumann, B.C. Kondratieff, C.R. Nelson, B.J. Sargent, $20 \hat{\jmath}, 15$ 우 (BYUC). Gila Co., Christopher Creek, Hwy 260, Christopher Creek Campground, 34.3110, -111.0244, 13 June 1974, B. Stark, 1ठ, 1 q (specimens from spider web, BPSC). Tonto Creek, above junction of Horton Creek, Upper Tonto Creek Campground, 34.3235, -111.2924, 14 January 1984, R.W. Baumann, C.R. Nelson, $18{ }^{\wedge}, 14$ 우 (BYUC). Graham Co., Twilight Creek, Turkey Flat, Pinaleño Mountains, 32.6310, 109.8187, 2 March 1984, C.R. Nelson, $3{ }^{\jmath}$, 1 우 (BYUC). Mohave Co., Wheeler Spring, Hualapai Mountain Park, Pine Basin Area, near Kingman, 35.0850, 113.8759, 20 January 1984, R.W. Baumann, C.R. Nelson, 28ㅅ, 13 (BYUC). Pima Co., Bear Canyon Creek, Bear Canyon Campground, Santa Catalina Mountains, 32.3276, -110.7328, 19 January 1984, R.W. Baumann, C.R. Nelson, 14へ̂, 10 (BYUC). Santa Cruz Co., Gardner Creek, Santa Rita Mountains, 31.8258, 110.7734, 17 January 1984, R.W. Baumann, C.R. Nelson, 9ત, 2 우 (BYUC).Yavapai Co., Lynx Creek, above Lynx Lake, near Prescott, 34.5145, -112.3812, 20 January 1988, R.W. Baumann, C.R. Nelson, $4 \hat{\circ}, 9$ 우 (BYUC).

\begin{tabular}{|l|l|l|l|l|l|l|l|}
\hline Table 2. Epiproct and $7^{\text {th }}$ tergal process characteristics for Arizona specimens of Arsapnia decepta. \\
\hline County & \multicolumn{1}{|c|}{ Site } & $\begin{array}{l}\text { Epiproct } \\
\text { Length } \mu \mathrm{mm}\end{array}$ & $\begin{array}{l}\text { Epiproct } \\
\text { Width } \mu \mathrm{m}\end{array}$ & $\begin{array}{l}\text { \# Left } \\
\text { Setae }\end{array}$ & $\begin{array}{l}\text { \# Right } \\
\text { Setae }\end{array}$ & $\begin{array}{l}\text { T7 } \\
\text { Process } \mu \mathrm{m}\end{array}$ & $\begin{array}{l}\text { Length/ } \\
\text { Width Ratio }\end{array}$ \\
\hline Cochise & $\begin{array}{l}\text { Cave Creek } \\
\text { SWRS }(\mathrm{n}=1)\end{array}$ & 478 & 239 & 20 & 20 & 229 & 2.00 \\
\hline Cochise & $\begin{array}{l}\text { Carr Canyon } \\
(\mathrm{n}=1)\end{array}$ & 541 & 245 & 30 & 25 & - & 2.21 \\
\hline Cochise & $\begin{array}{l}\text { Rucker Creek } \\
(\mathrm{n}=1)\end{array}$ & 462 & 248 & 27 & 25 & - & 1.86 \\
\hline Coconino & $\begin{array}{l}\text { Oak Creek } \\
(\mathrm{n}=1)\end{array}$ & 578 & 278 & 27 & 29 & - & 2.08 \\
\hline Gila & $\begin{array}{l}\text { Christopher Creek } \\
(\mathrm{n}=1)\end{array}$ & 459 & 265 & 15 & 16 & 172 & 1.73 \\
\hline Gila & $\begin{array}{l}\text { Tonto Creek } \\
(\mathrm{n}=2)\end{array}$ & $511-524$ & $260-263$ & $23-27$ & $24-28$ & - & $1.95-2.00$ \\
\hline Graham & $\begin{array}{l}\text { Turkey Creek } \\
(\mathrm{n}=1)\end{array}$ & 524 & 247 & 28 & 28 & - & 2.12 \\
\hline Mohave & $\begin{array}{l}\text { Wheeler Spring } \\
(\mathrm{n}=2)\end{array}$ & 561 & 256 & $20-28$ & $18-24$ & - & 2.19 \\
\hline Pima & $\begin{array}{l}\text { Bear Canyon } \\
(\mathrm{n}=1)\end{array}$ & 549 & 262 & 31 & 34 & 194 & 2.09 \\
\hline Santa Cruz & $\begin{array}{l}\text { Gardner Creek } \\
(\mathrm{n}=2)\end{array}$ & $438-452$ & 200 & $18-22$ & $20-26$ & - & $2.19-2,29$ \\
\hline Yavapai & $\begin{array}{l}\text { Lynx Creek } \\
(\mathrm{n}=1)\end{array}$ & 511 & 263 & 26 & 30 & - & 1.94 \\
\hline
\end{tabular}



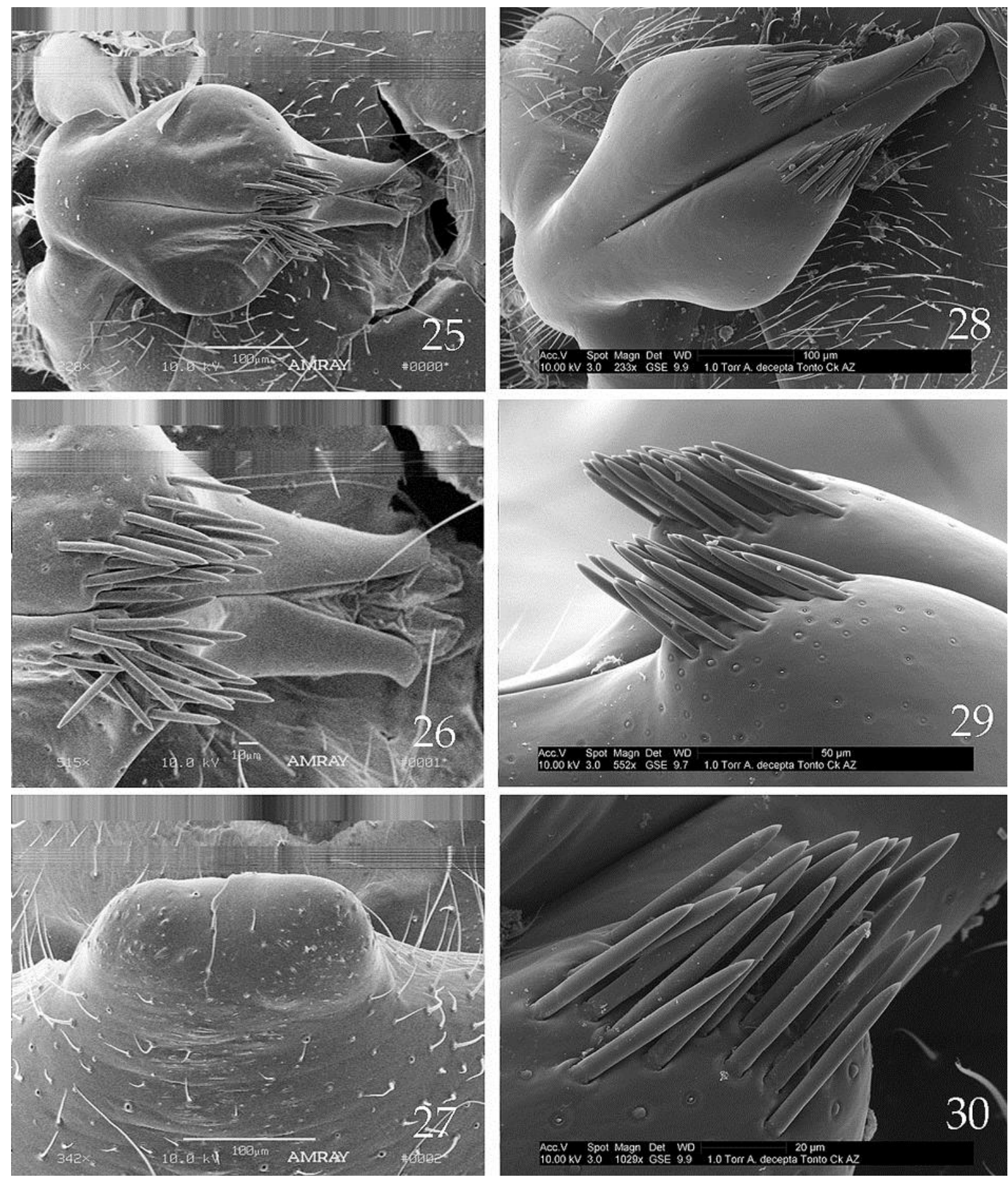

Figs. 25-30. Arsapnia decepta male reproductive structures. 25-27. Christopher Creek, Gila Co., Arizona (damaged). 28-30. Tonto Creek, Gila Co., Arizona. 25. Epiproct, dorsal. 26. Epiproct apex and setal spine clusters, dorsal. 27. $7^{\text {th }}$ tergal process, anterodorsal. 28. Epiproct, dorsal. 29. Epiproct bulbs and setal spine clusters, oblique lateral. 30 . Right setal cluster, posterodorsal. 

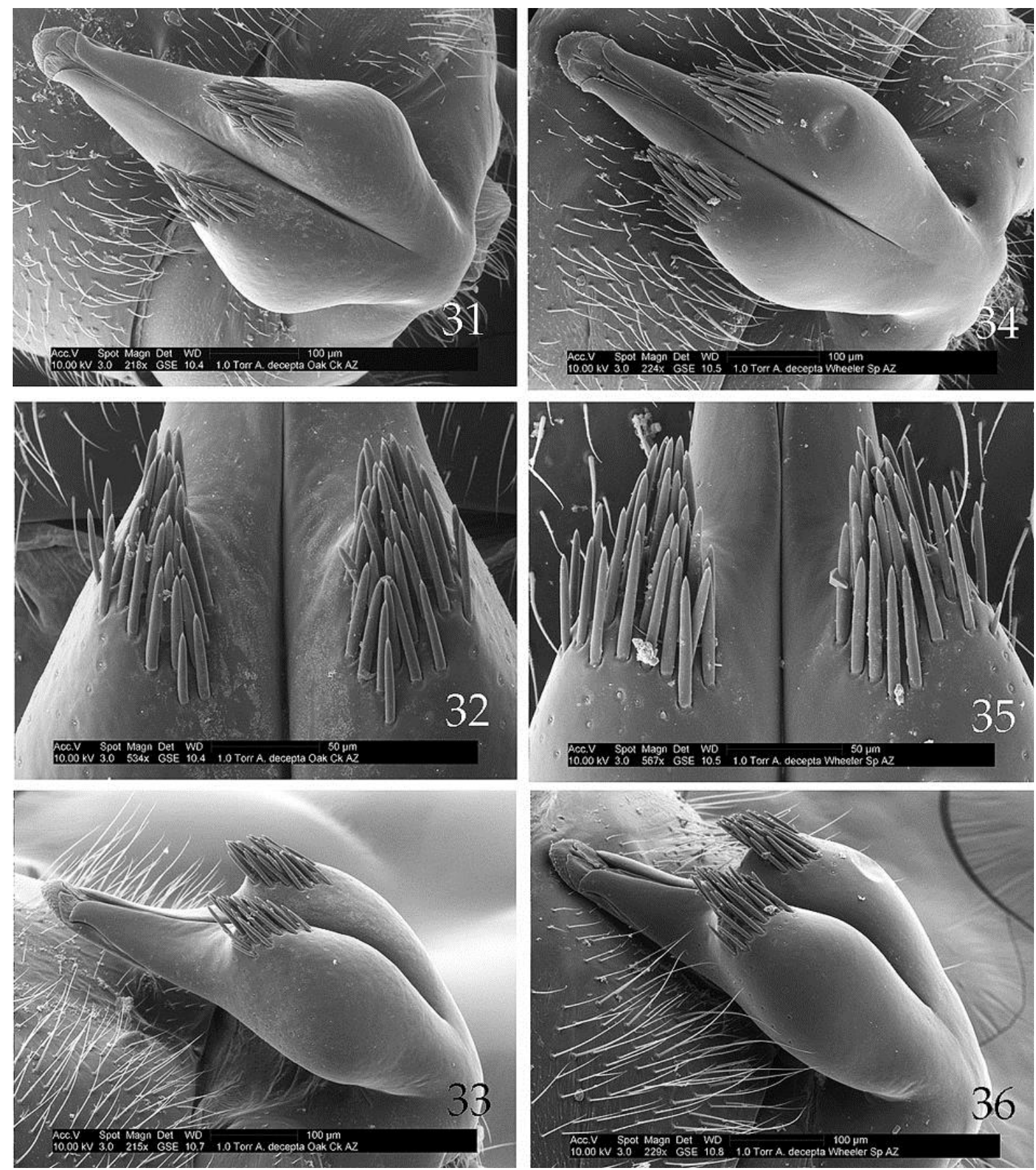

Figs. 31-36. Arsapnia decepta male reproductive structures. 31-33. West Fork Oak Creek, Coconino Co., Arizona, 34-36. Wheeler Spring, Hualapi Mountain Park, Mohave Co., Arizona. 31. Epiproct, dorsal. 32. Epiproct setal spine clusters, dorsal. 33. Epiproct, dorsolateral. 34. Epiproct, dorsal. 35. Epiproct setal spine clusters, dorsal. 36. Epiproct, dorsolateral. 
Baumann, R.W., and B.P. Stark 2017. Variation in the Epiproct of Arsapnia decepta Banks, 1897 (Plecoptera: Capniidae), with Comments on Arsapnia coyote (Nelson \& Baumann 1987). Illiesia, 13(01):1-21. https://doi.org/10.25031/2017/13.01

Male epiproct $(n=14)$. Length $438-578 \mu \mathrm{m}$, width 200-278 $\mu \mathrm{m}$ (Table 2). Shape and general structure similar to those of other populations examined (Figs. 13, 18, 19, 22, 33, 36, 39, 42, 45). Setal spines in clusters of 15-34 (Figs. 14-17, 20-21, 23-26, 28-32, 3435, 37-38, 40-41, 43-44). Neck width 100-144 $\mu \mathrm{m}$ (Figs. 11, 14, 16, 20, 23, 28, 31, 34, 37, 40, 43).

Tergal process $(n=3)$. Tergum 7 bears a broad, sometimes mesally notched, dorsal process (Figs. 13, 19, 22, 27), and tergum 6 bears a smaller mesal process on one specimen from the Chiricahua Mountains. Dorsolateral margins bearing a few knob-like scales. Width across anterior margin $172-229 \mu \mathrm{m}$.

Comments. The first Arizona records for A. decepta are attributed to Jewett (1966) (as C. barbata), and Stewart et al. (1974) show plots of six sites (also as C. barbata) in five Arizona counties (Apache, Cochise, Gila, Pima, and Yavapai). Our specimens represent populations from eight counties (Cochise, Coconino, Gila, Graham, Mohave, Pima, Santa Cruz and Yavapai) and the following natural regions: the Mogollon Rim, Oak Creek Canyon and Yavapai Hills in central Arizona, the Hualapai Mountains in west central Arizona, and the Chiricahua,
Huachuca, Pinaleño, Santa Catalina and Santa Rita Mountains in southeastern Arizona. Nelson \& Baumann (1987a) provided SEM micrographs for specimens collected in Ramsey Canyon, Huachuca Mountains, Cochise Co., Arizona.

This sample of Arizona specimens includes males with the three longest epiprocts $(578 \mu \mathrm{m}$, Oak Creek Canyon; $561 \mu \mathrm{m}$, Wheeler Spring; $549 \mu \mathrm{m}$, Bear Canyon) in the entire sample, and all fell within the $95 \%$ confidence interval $(247.2 \pm 13.1 \mu \mathrm{m})$ in epiproct width. In addition, the 9 highest counts of setal spines per cluster (27-34) were recorded from these Arizona specimens. The greatest number of setal spines (31 left, 34 right) were recorded on a Bear Canyon (Pima Co.) specimen.

\section{Nevada Population}

(Figs. 46-48)

Material examined. USA: Nevada: Clark Co., Deer Creek, Deer Creek Picnic Area, Hwy 158, Spring Mountains, near Las Vegas, 36.3144, -115.6212, 21 January 1984, R.W. Baumann, C.R. Nelson, $1 \hat{\delta}, 3$ ㅇ (BYUC).

Table 3. Epiproct and $7^{\text {th }}$ tergal process characteristics for Nevada specimens of Arsapnia decepta.

\begin{tabular}{|l|l|l|l|l|l|l|l|}
\hline County & Site & $\begin{array}{l}\text { Epiproct } \\
\text { Length } \mu \mathrm{m}\end{array}$ & $\begin{array}{l}\text { Epiproct } \\
\text { Width } \mu \mathrm{m}\end{array}$ & $\begin{array}{l}\text { \# Left } \\
\text { Setae }\end{array}$ & $\begin{array}{l}\text { \# Right } \\
\text { Setae }\end{array}$ & $\begin{array}{l}\text { T7 } \\
\text { Process } \mu \mathrm{m}\end{array}$ & $\begin{array}{l}\text { Length/ } \\
\text { Width Ratio }\end{array}$ \\
\hline Clark & $\begin{array}{l}\text { Deer Creek } \\
(\mathrm{n}=1)\end{array}$ & 379 & 157 & 31 & 30 & 185 & 2.41 \\
\hline
\end{tabular}

Male epiproct $(\mathrm{n}=1)$. Length $379 \mu \mathrm{m}$, width $157 \mu \mathrm{m}$ (Table 3). Shape and general structure similar to those of other specimens, but smaller and with higher setal spine counts. Setal spines in clusters of 30-31 located anterior of midlength and on either side of median groove (Figs. 46-48). Neck width 135 $\mu \mathrm{m}$.

Tergal process. Tergum 7 bearing a broad dorsal process, $185 \mu \mathrm{m}$ in width; dorsal margin concave; lateral prominences covered with small scale-like structures.
Comments. The Spring Mountains represent the only known site where $A$. decepta specimens have been collected in Nevada (Nelson \& Baumann 1989). We examined a single male specimen from Deer Creek at its junction with Hwy 158. As Figs. 46-48 and Tables 1-5 indicate, the epiproct of this specimen is shorter and more slender than other specimens examined, and also has one of the highest setal spine counts we encountered, second only to the Bear Canyon specimens from Arizona. 

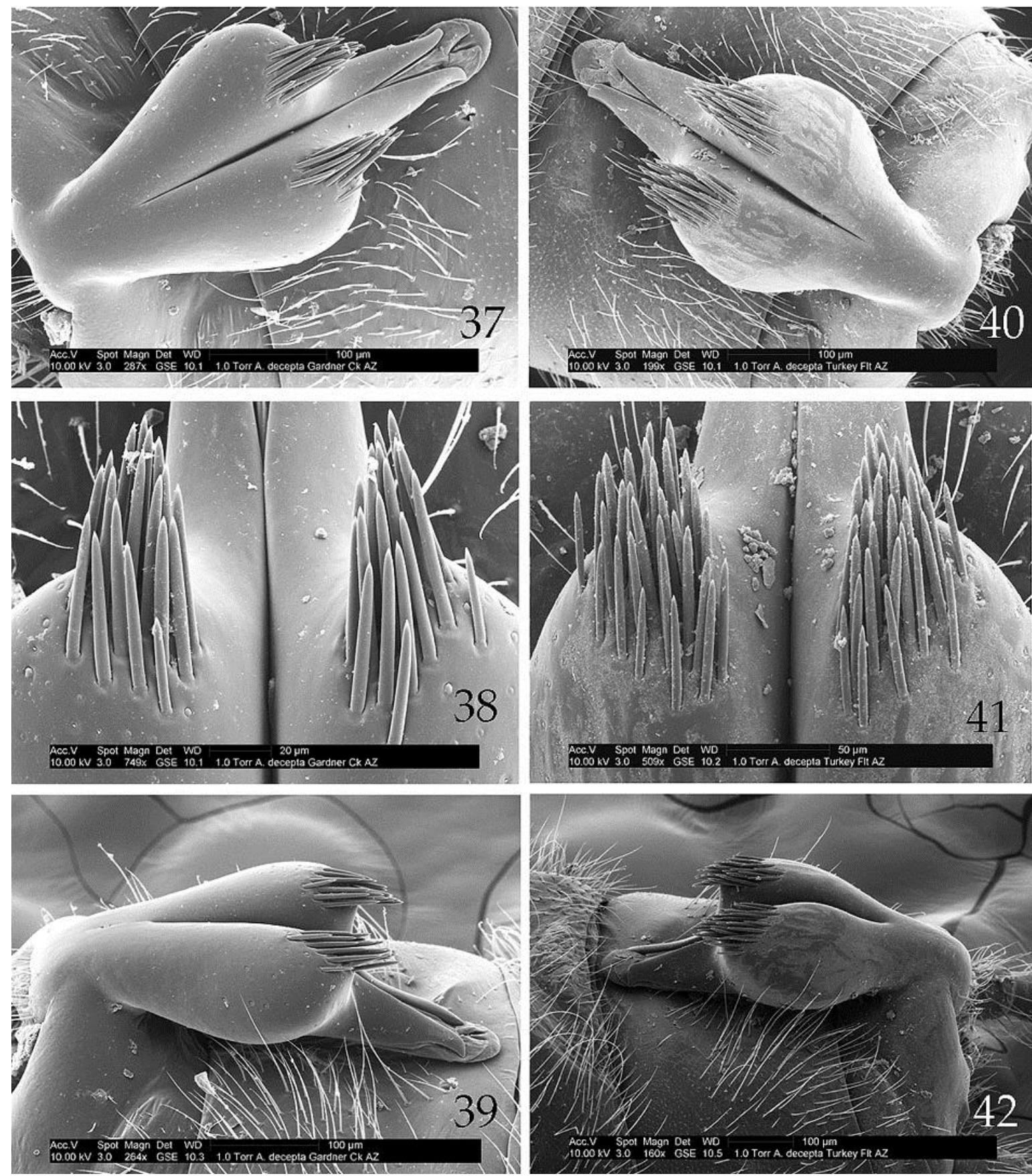

Figs. 37-42. Arsapnia decepta male reproductive structures. 37-39. Gardner Creek, Santa Rita Mountains, Santa Cruz Co., Arizona. 40-42. Twilight Creek, Pinaleño Mountains, Graham Co., Arizona. 37. Epiproct, dorsal. 38. Epiproct setal spine clusters, dorsal. 39. Epiproct, dorsolateral. 40. Epiproct, dorsal. 41. Epiproct setal spine clusters, dorsal. 42. Epiproct, dorsolateral. 

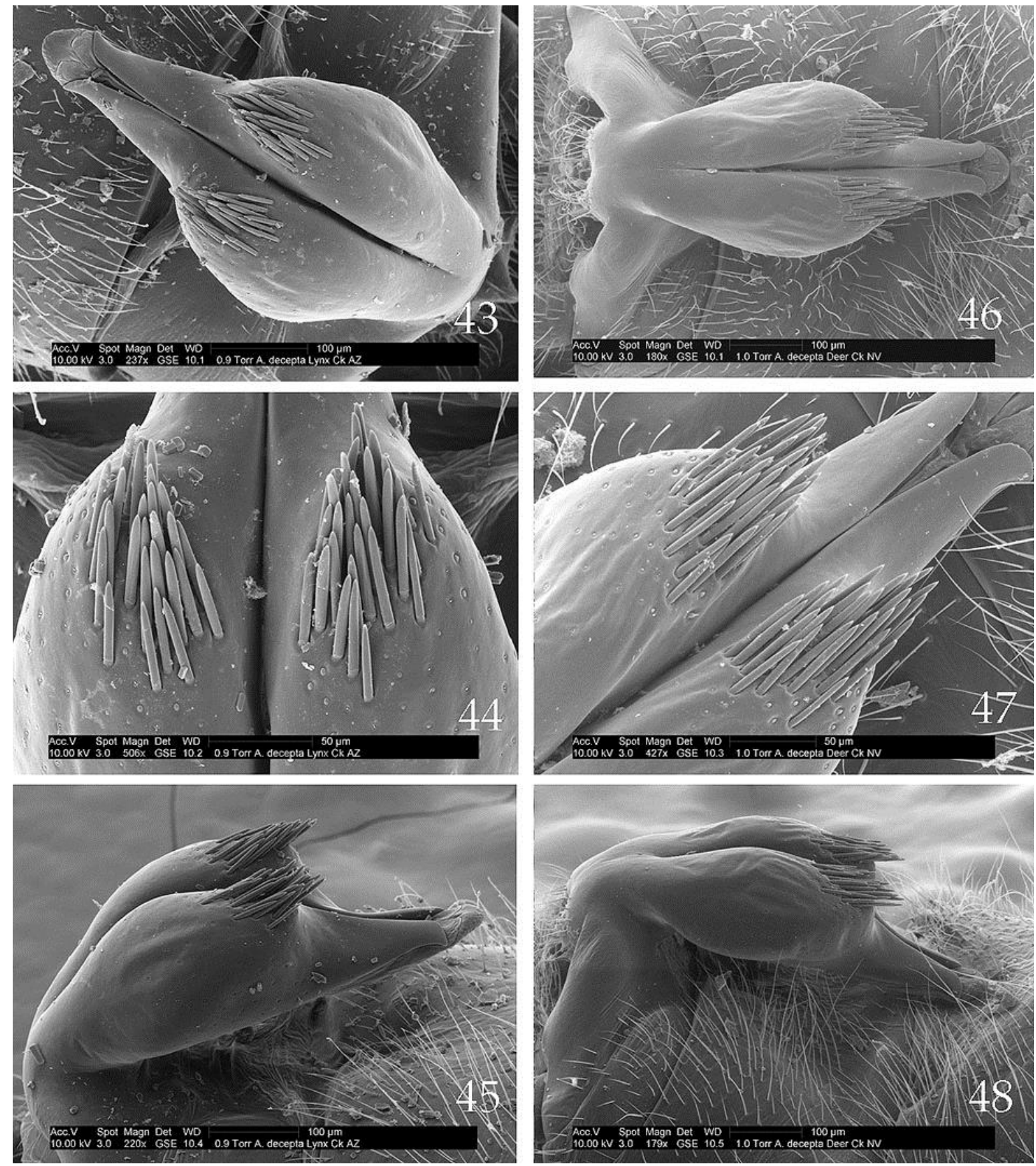

Figs. 43-48. Arsapnia decepta male reproductive structures. 43-45. Lynx Creek, Yavapai Co., Arizona. 46-48. Deer Creek, Spring Mountains, Clark Co., Nevada. 43. Epiproct, dorsal. 44. Epiproct setal spine clusters, dorsal. 45. Epiproct, dorsolateral. 46. Epiproct, dorsal. 47. Epiproct setal spine clusters, dorsal. 48. Epiproct, dorsolateral. 
New Mexico Populations

(Figs. 49-60)

Material examined. USA: New Mexico: Catron Co., Leggett Canyon Creek, Leggett Canyon, Hwy 180, south of Luna, 33.7076, -108.8938, 13 January 1987, R.W. Baumann, B.C. Kondratieff, B.J. Sargent, 7ð, 1 우 (BYUC). Grant Co., Gila River, Hwy 180, south of Cliff, 32.9451, -108.6069, 13 January 1987, R.W. Baumann, B.C. Kondratieff, B.J. Sargent, $1 \hat{\delta}, 1$ 우 (BYUC). Lincoln Co., Eagle Creek, Rt. 532, south of Sierra Vista, 33.3925, -105.6885, 11 March 1996, B. Stark, 7ð, 5 우 (BPSC). Lincoln Co., Rio Bonito, below Bonito Lake, Bonito Lake Road, 33.4516, -105.6953, 11 March 1996, B. Stark, 31 ${ }^{\AA}, 37$ ㅇ (BPSC).

Male epiproct $(n=12)$. Length $412-562 \mu \mathrm{m}$, width 180-269 $\mu \mathrm{m}$ (Table 4). Shape and general structure as in other populations. Setal spines in clusters of 1021 , located near midlength and on either side of median groove (Figs. 51, 54, 55-60). Neck width 105$130 \mu \mathrm{m}$.

Tergal process. Bearing a slight notch on the posteromedian margin (Fig. 49-52, 57-58), and with lateral margins moderately convergent. Dorsolateral lobes on either side of notch covered with conical tubercles. Width of process across anterior margin 100-192 $\mu \mathrm{m}$.

Comments. Arsapnia decepta has been reported (as Capnia decepta or C. barbata) from numerous sites in 12 counties of New Mexico (Jacobi \& Baumann 1983, Jacobi \& Cary 1986, Jacobi \& Cary 1996, Jacobi et al. 2005). The collecting sites listed above include specimens from two streams that are in the Rio Hondo drainage in Lincoln Co. Despite the close proximity of these two sites, the epiprocts from Eagle Creek specimens are shorter and narrower than those from Rio Bonito (Table 4), but the Eagle Creek specimens have an apex that is longer and narrower than those from Rio Bonito (compare Figs. 50 with 53, and 51 with 54). Among the specimens illustrated with micrographs (Figs. 49-54), the Eagle Creek specimens show asymmetrical setal spine counts of 10-17 left and 10-15 right (Table 4). The counts for both of these ranges are also the lowest observed for any A. decepta population. This could be related to the smaller epiproct size for Eagle Creek specimens, or may simply reflect the small sample size for all populations examined. As indicated in Table 4, epiproct size and setal spine counts for Catron and Grant county specimens are more similar to those from adjacent states than to the Eagle Creek specimens.

Table 4. Epiproct and $7^{\text {th }}$ tergal process characteristics for New Mexico specimens of Arsapnia decepta.

\begin{tabular}{|l|l|l|l|l|l|l|l|}
\hline County & \multicolumn{1}{|c|}{ Site } & $\begin{array}{l}\text { Epiproct } \\
\text { length } \mu \mathrm{m}\end{array}$ & $\begin{array}{l}\text { Epiproct } \\
\text { Width } \mu \mathrm{m}\end{array}$ & $\begin{array}{l}\text { \# Left } \\
\text { Setae }\end{array}$ & $\begin{array}{l}\text { \# Right } \\
\text { Setae }\end{array}$ & $\begin{array}{l}\text { T7 } \\
\text { Process } \mu \mathrm{m}\end{array}$ & $\begin{array}{l}\text { Length/ } \\
\text { Width Ratio }\end{array}$ \\
\hline Catron & $\begin{array}{l}\text { Leggett } \\
\text { Canyon Creek } \\
(\mathrm{n}=1)\end{array}$ & 562 & 269 & 17 & 21 & - & 2.08 \\
\hline Grant & $\begin{array}{l}\text { Gila River } \\
(\mathrm{n}=1)\end{array}$ & 468 & 237 & - & 20 & 168 & 1.97 \\
\hline Lincoln & $\begin{array}{l}\text { Eagle Creek } \\
(\mathrm{n}=3)\end{array}$ & $412-413$ & $180-200$ & $10-17$ & $10-15$ & $146-168$ & $2.06-2.29$ \\
\hline Lincoln & $\begin{array}{l}\text { Rio Bonito } \\
(\mathrm{n}=7)\end{array}$ & $436-483$ & $217-244$ & $12-19$ & $12-18$ & $100-192$ & $1.84-2.02$ \\
\hline
\end{tabular}



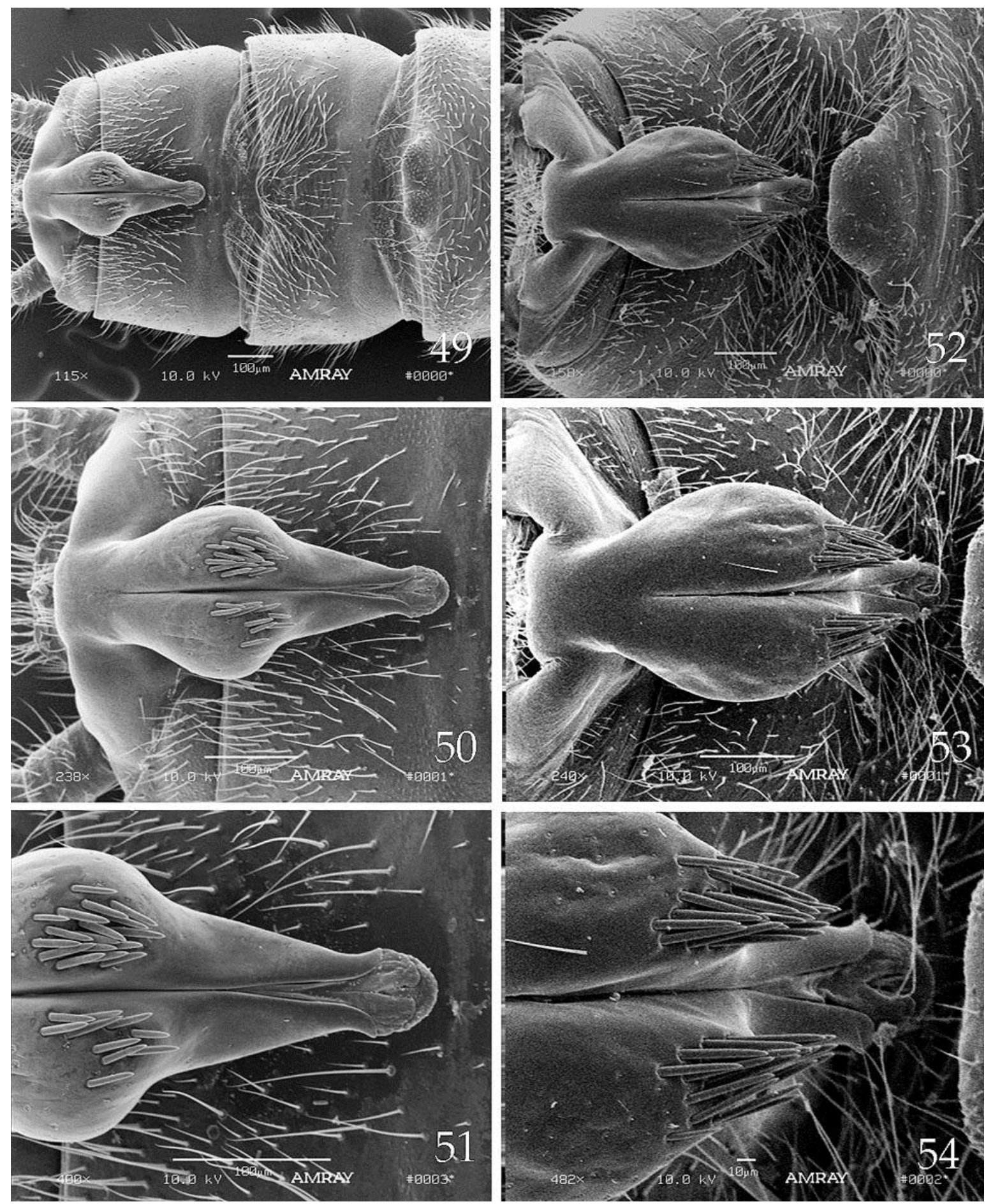

Figs. 49-54. Arsapnia decepta male reproductive structures. 49-51. Eagle Creek, Lincoln Co., New Mexico. 5254. Rio Bonito, Lincoln Co., New Mexico. 49. Abdominal terga 7-10. 50. Epiproct, dorsal. 51. Epiproct apex and setal spine clusters. 52. Abdominal terga 7-10. 53. Epiproct, dorsal. 54. Epiproct apex and setal spine clusters. 

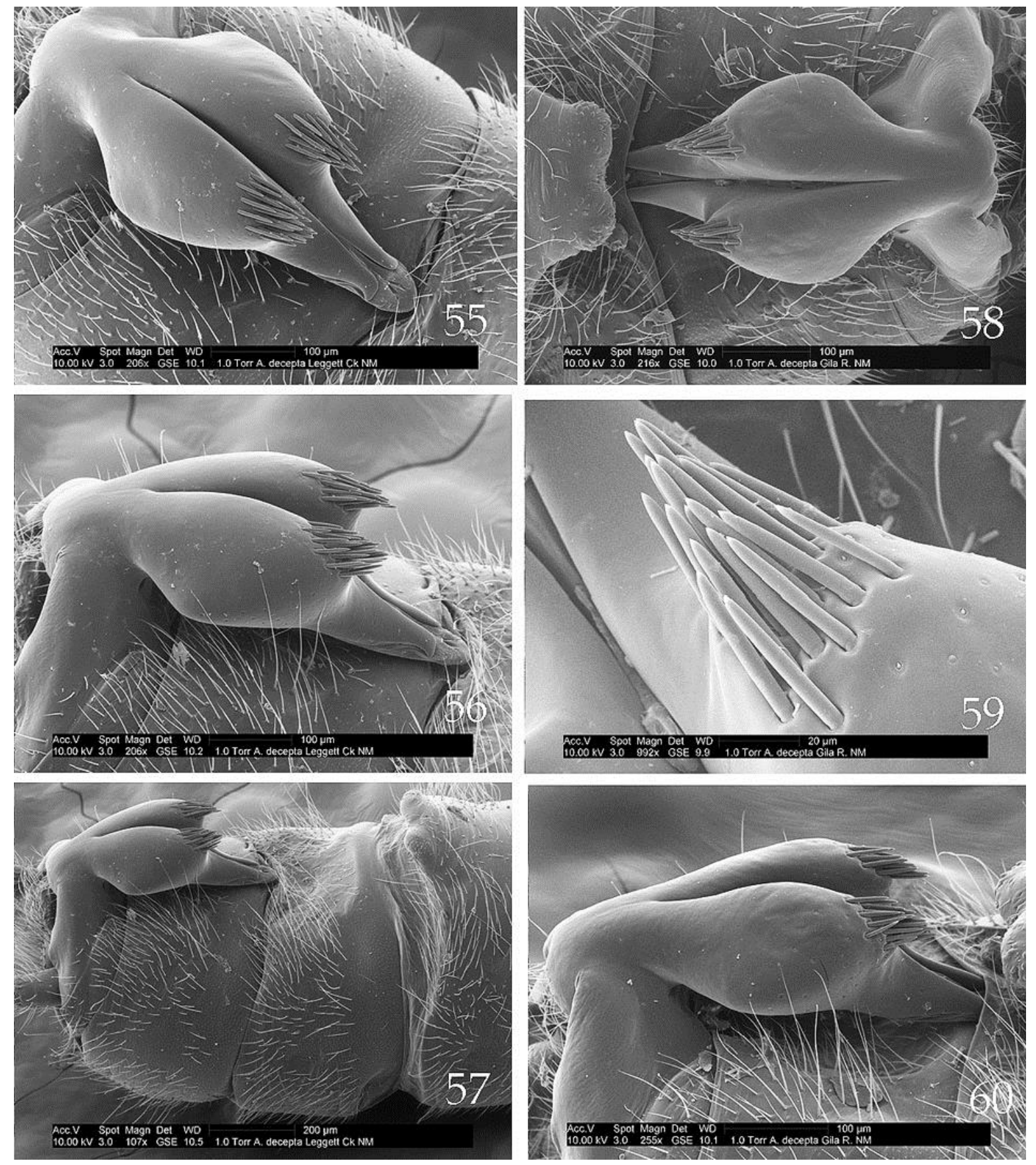

Figs. 55-60. Arsapnia decepta male reproductive structures. 55-57. Leggett Canyon Creek, Catron Co., New Mexico. 58-60. Gila River, Grant Co., New Mexico. 55. Epiproct, dorsal. 56. Epiproct, dorsolateral. 57. Abdominal segments 7-10, lateral. 58. Epiproct and $7^{\text {th }}$ tergal process, dorsal. 59. Right setal cluster, dorsal. 60. Epiproct, dorsolateral. 
Mexico Populations

(Figs. 61-66)

Material examined. MEXICO: Baja California: Arroyo La Corona, Sierra San Pedro Martir National Park, 16 January 1988, R.W. Baumann, B.C. Kondratieff, C.R. Nelson, B.J. Sargent, 1 pharate $\delta^{\lambda}$ larva (dissected), 4 , 4 larvae. Chihuahua: Cañon de Agua, Bowman Ranch, near Colonia Juarez, 22 January 1987, B.C. Kondratieff, B.J. Sargent, T. Bowman, 2へ, 4 ㅇ (BYUC).

Male epiproct $(\mathrm{n}=2)$. Length not estimated due to specimen condition and orientation (Figs. 61, 64; Table 5). Width $238 \mu \mathrm{m}$. Shape and general features similar to other specimens examined. Setal spines in clusters of 13-18 (Table 5, Figs. 61-66). Neck width $93 \mu \mathrm{m}$ (Fig. 64).

Tergal process. Not visible in available figures.

Comments. Only two male specimens were available for SEM study (Table 5) including a pharate male collected in Baja California and dissected by B.C. Kondratieff from the larval skin. Unfortunately, only six SEM images were prepared due to specimen condition and orientation (Figs. 6166). Despite the poor condition and orientation of these specimens, their epiprocts display the same general shape and the typical setal spine clusters found among other populations. The Baja California population should be re-examined with molecular data and with a more complete set of morphological data, and compared with California specimens of $A$. coyote when suitable material is available. The epiproct of $A$. coyote is similar, but distinct from A. decepta morphologically (see Figs. 67-72), and Heinold et al. (2014) also support recognition of $A$. coyote as a valid species based on molecular barcode data. The Chihuahua population exhibits a narrow neck width $(93 \mu \mathrm{m})$ more consistent with $A$. coyote, but with respect to epiproct width, the specimen is more similar to $A$. decepta.

Table 5. Epiproct and $7^{\text {th }}$ tergal process characteristics for Mexican specimens of Arsapnia decepta.

\begin{tabular}{|l|l|l|l|l|l|l|l|}
\hline Mexican State & \multicolumn{1}{|c|}{ Site } & $\begin{array}{l}\text { Epiproct } \\
\text { Length } \mu \mathrm{m}\end{array}$ & $\begin{array}{l}\text { Epiproct } \\
\text { Width } \mu \mathrm{m}\end{array}$ & $\begin{array}{l}\text { \# Left } \\
\text { Setae }\end{array}$ & $\begin{array}{l}\text { \# Right } \\
\text { Setae }\end{array}$ & $\begin{array}{l}\text { T7 } \\
\text { Process } \mu \mathrm{m}\end{array}$ & $\begin{array}{l}\text { Length/ } \\
\text { Width Ratio }\end{array}$ \\
\hline Baja California & $\begin{array}{l}\text { Arroyo La Corona } \\
(\mathrm{n}=1)\end{array}$ & - & - & 15 & 13 & - & - \\
\hline Chihuahua & $\begin{array}{l}\text { Cañon de Agua } \\
(\mathrm{n}=1)\end{array}$ & - & 238 & 18 & 15 & 157 & - \\
\hline
\end{tabular}

\section{Arsapnia coyote (Nelson \& Baumann) Coyote Snowfly}

(Figs. 67-72)

Capnia coyote Nelson \& Baumann, 1987b:487. Holotype $\hat{\jmath}$, (United States National Museum), Little Rock Creek, Cooper Canyon Campground, San Gabriel Mountains, Los Angeles Co., California http://lsid.speciesfile.org/urn:lsid:Plecoptera.speciesfile.org: TaxonName:4978

Arsapnia coyote: Murányi, Gamboa \& Orci, 2014:14 http://lsid.speciesfile.org/urn.lsid:Plecoptera.speciesfile.org: TaxonName:465452
Material examined. USA: California: Los Angeles Co., Little Rock Creek, Cooper Canyon Campground, San Gabriel Mountains, 31 March 1981, R.W. Baumann, J. Stanger, $3 \hat{\circ}$ (BYUC). San Bernardino Co., East Fork of West Fork before Mohave River above Silverwood Lake, 9 January

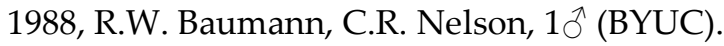

Male epiproct $(n=2)$. Length $575-618 \mu \mathrm{m}$, width at midlength 223-236 $\mu \mathrm{m}$, Body of epiproct expanded into convex ear-like lobes near midlength (Figs. 67, 70). Width across neck 67-83 $\mu \mathrm{m}$. Shape and 

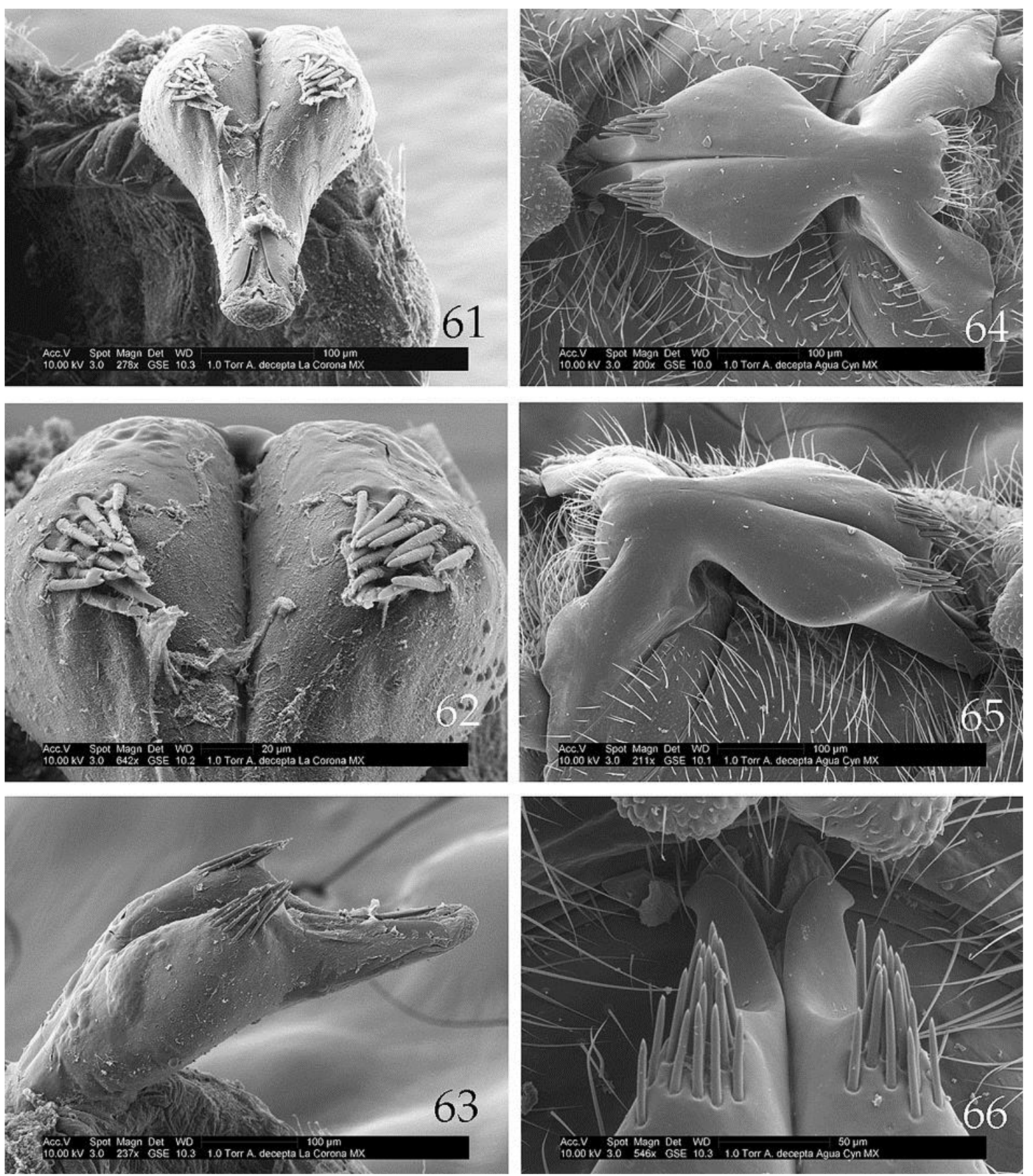

Figs. 61-66. Arsapnia decepta male reproductive structures, 61-63. Arroyo La Corona, Sierra San Pedro Martir National Park, Baja California. 64-66. Cañon de Agua, Bowman Ranch, near Colonia Juarez, Chihuahua. 61. Epiproct, anterodorsal. 62. Epiproct, setal spine clusters, anterodorsal. 63. Epiproct, lateral. 64. Epiproct, dorsal. 65. Epiproct, oblique lateral. 66. Epiproct apex and setal spine clusters, dorsal. 

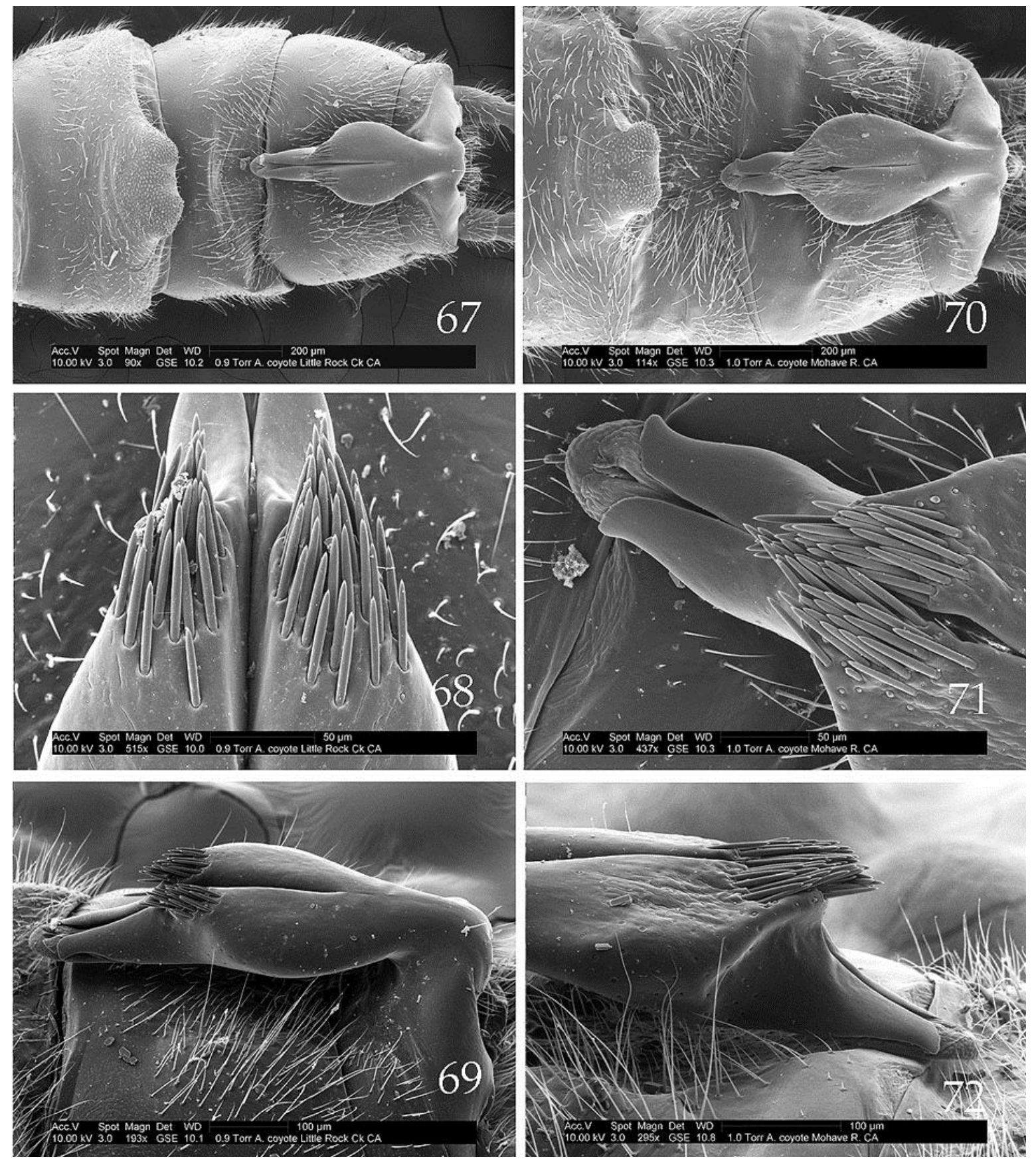

Figs. 67-72. Arsapnia coyote male reproductive structures. 67-69. Little Rock Creek, Los Angeles Co., California.70-72. Mohave River, San Bernardino Co., California. 67. Abdominal segments 7-10, dorsal. 68. Epiproct apex and setal spine clusters. 69. Epiproct, oblique lateral. 70. Abdominal segments 7-10, dorsal. 71. Epiproct apex and setal spine clusters. 72. Epiproct apex, lateral. 
Baumann, R.W., and B.P. Stark 2017. Variation in the Epiproct of Arsapnia decepta Banks, 1897 (Plecoptera: Capniidae), with Comments on Arsapnia coyote (Nelson \& Baumann 1987). Illiesia, 13(01):1-21. https://doi.org/10.25031/2017/13.01

general structure similar to those of populations of A. decepta examined. Left side setal spines in clusters of 25-28, and 28-32 on the right.

Tergal process $(n=2)$. Bearing a slight to moderate notch of posteromedian margin (Figs. 67-70). Dorsolateral lobes bearing patches of small conical tubercles. Width of process across anterior margin 200-280 $\mu \mathrm{m}$.

Comments. Arsapnia coyote is presently considered an endemic to southern California and the sister species of A. decepta (Nelson \& Baumann 1987b). The two species overlap in many morphological features including epiproct width and setal spine counts, however two of the three longest epiprocts among specimens studied are of this species, and the epiproct length/width ratios (2.58 and 2.61 respectively, Table 6) for these two specimens are the highest observed. Nelson \& Baumann (1987b) distinguished the two species, in part, by virtue of a flatter dorsal epiproct surface and smaller epiproct depth (compare Figs. 69 and 72 with Figs. 4 and 18). Recently, Heinold et al. (2014) supported recognition of $A$. coyote as a distinct species based on their report of an average genetic divergence of $1.9 \%$ between males of $A$. coyote and A. decepta.

\section{Table 6. Epiproct and $7^{\text {th }}$ tergal process characteristics for California specimens of Arsapnia coyote.}

\begin{tabular}{|c|l|l|l|l|l|l|l|}
\hline County & \multicolumn{1}{|c|}{ Site } & $\begin{array}{l}\text { Epiproct } \\
\text { Length } \mu \mathrm{m}\end{array}$ & $\begin{array}{l}\text { Epiproct } \\
\text { Width } \mu \mathrm{m}\end{array}$ & $\begin{array}{l}\text { \# Left } \\
\text { Setae }\end{array}$ & $\begin{array}{l}\text { \# Right } \\
\text { Setae }\end{array}$ & $\begin{array}{l}\text { T7 } \\
\text { Process } \mu \mathrm{m}\end{array}$ & $\begin{array}{l}\text { Length/ } \\
\text { Width Ratio }\end{array}$ \\
\hline Los Angeles & $\begin{array}{l}\text { Little Rock } \\
\text { Creek (n=1) }\end{array}$ & 575 & 223 & 28 & 32 & 280 & 2.58 \\
\hline San Bernardino & $\begin{array}{l}\text { Mohave } \\
\text { River (n=1) }\end{array}$ & 618 & 236 & 25 & 28 & 200 & 2.61 \\
\hline
\end{tabular}

\section{DISCUSSION}

Arsapnia decepta is a common winter-emerging species of the Southwestern United States and adjacent areas of Mexico. Many populations exist in relative isolation, consequently significant variation in male reproductive structures occurs. Much of this variation centers around epiproct length, width and setal spine counts for the left and right epiproct clusters. Setal spine counts for left side clusters range from 10 to 31 and right side clusters range from 10 to 34 in our samples. The lowest counts occurred among three individuals from Eagle Creek and Rio Bonito, New Mexico with clusters of 23, 25 and 25 total setal spines $(10,13 ; 12,13 ; 10,15)$, and other relatively low counts were observed for Buckhorn Creek, Colorado (14,12), and Cedar Creek, Colorado $(14,14)$. The highest setal spine counts were from Bear Canyon, Arizona $(31,34)$ and Deer Creek, Nevada $(31,30)$ specimens. Total setal spine counts ranged from 40-65 for Arizona specimens, 26-43 for Colorado specimens, and 23-35 for New Mexico specimens. Arsapnia coyote setal spine counts (53-60) were within the range of Arizona A. decepta specimens.
Arsapnia decepta epiprocts ranged from 379-578 $\mu \mathrm{m}$ in length (mean $=487.9 \pm 19.4$ ), but seven of the eight longest epiprocts were from Arizona (524-578 $\mu \mathrm{m})$ or Colorado (525-528 $\mu \mathrm{m})$. Only two of the New Mexico specimens had epiproct lengths greater than $500 \mu \mathrm{m}$. The epiproct lengths for the two specimens of $A$. coyote we examined were 575 and $618 \mu \mathrm{m}$. These lengths exceeded all but one of the A. decepta specimens. The two species overlap in setal spine counts and in epiproct length, however the epiproct depth character noted by Nelson \& Baumann (1987b, 1989), although not easily measured with SEM, appears to be a reliable morphological character for distinguishing $A$. decepta and A. coyote. This paper is not a revision or a complete geographical study of $A$. decepta and $A$. coyote as presently known. Instead it is an overview of specimens from states in the United States and Mexico where specimens have been selected that show variation in epiproct structure. Complete museum records of these species are not recorded and only those specimens that were used for SEM study are included. Thus, the inclusion of a map showing the complete geographical distributions of 
Baumann, R.W., and B.P. Stark 2017. Variation in the Epiproct of Arsapnia decepta Banks, 1897 (Plecoptera: Capniidae), with Comments on Arsapnia coyote (Nelson \& Baumann 1987). Illiesia, 13(01):1-21. https://doi.org/10.25031/2017/13.01

these species is left for zoographical studies in the future.

\section{ACKNOWLEDGEMENTS}

We appreciate Boris Kondratieff, Colorado State University, C. Riley Nelson, Brigham Young University, and B.J. Sargent for their assistance in collecting Arsapnia specimens. We also thank Boris for rearing the mature nymphs from Baja California, for the loan of specimens from the Colorado Front Range, and for help in locating references. We also thank Denise Mason for her assistance in preparing micrographs for specimens from Redstone Canyon, Colorado and Rio Bonito, New Mexico.

\section{REFERENCES}

Banks, N. 1897. New North American Neuropteroid Insects. Transactions of the American Entomological Society, 24:21-31.

Claassen, P.W. 1924. New species of North American Capniidae (Plecoptera). Canadian Entomologist, 56:43-48, 54-57.

DeWalt, R.E., M.D. Maehr, U. Neu-Becker, \& G. Stueber. 2017. Plecoptera species file online. Version 5.0/5.0. Retrieved 17 February 2017.

Frison, T.H. 1944. Three new species of Capnia from Colorado (Plecoptera: Capniidae). Transactions of the American Entomological Society, 69:151157.

Heinold, B.D., B.A. Gill, T.P. Belcher, \& C.J. Verdone. 2014. Discovery of new populations and DNA barcoding of the Arapahoe snowfly Arsapnia arapahoe (Plecoptera: Capniidae). Zootaxa, 3866:131-137.

Jacobi, G.Z. \& R.W. Baumann. 1983. Winter stoneflies (Plecoptera) of New Mexico. Great Basin Naturalist, 43:585-591.

Jacobi, G.Z. \& S.J. Cary. 1986. New records of winter stoneflies (Plecoptera) from southwestern New Mexico, with notes on habitat preferences and zoogeographic origins. Southwestern Naturalist, 31:503-510.

Jacobi, G.Z. \& S.J. Cary. 1996. Winter stoneflies (Plecoptera) in seasonal habitats in New Mexico, USA. Journal of the North American Benthological Society, 15:690-699.

Jacobi, G.Z., S.J. Cary, \& R.W. Baumann. 2005. An updated list of the stoneflies (Plecoptera) of New Mexico, U.S.A. Entomological News, 116:29-34.

Jewett, S.G., Jr. 1966. Some species of Capnia from western North America (Plecoptera). Wasmann Journal of Biology, 24:101-108.

Kondratieff, B.C. \& R.W. Baumann. 2002. A review of the stoneflies of Colorado with description of a new species of Capnia (Plecoptera: Capniidae). Transactions of the American Entomological Society, 128:385-401.

Murányi, D., M. Gamboa, \& K.M. Orci. 2014. Zwicknia gen. n., a new genus for the Capnia bifrons species group, with descriptions of three new species based on morphology, drumming signals and molecular genetics, and a synopsis of the West Palearctic and Nearctic genera of Capniidae (Plecoptera). Zootaxa, 3812:1-82.

Nelson, C.R. \& R.W. Baumann. 1987a. Scanning electron microscopy for the study of the winter stonefly genus Capnia (Plecoptera: Capniidae). Proceedings of the Entomological Society of Washington, 89:51-56.

Nelson, C.R. \& R.W. Baumann. 1987b. New winter stoneflies of the genus Capnia with notes and an annotated checklist of the Capniidae of California (Plecoptera: Capniidae). Entomography, 5:485-521.

Nelson, C.R. \& R.W. Baumann. 1989. Systematics and distribution of the winter stonefly genus Capnia (Plecoptera: Capniidae) in North America. Great Basin Naturalist, 49:289-363.

Sargent, B.J., R.W. Baumann, \& B.C. Kondratieff. 1991. Zoogeographic affinities of the Nearctic stonefly (Plecoptera) fauna of Mexico. Southwestern Naturalist, 36:323-331.

Stark, B.P., B.R. Oblad, \& A.R. Gaufin. 1973. An annotated list of the stoneflies (Plecoptera) of Colorado. Part 1. Entomological News, 84:269277.

Stewart, K.W., R.W. Baumann, \& B.P. Stark. 1974. The distribution and past dispersal of southwestern United States Plecoptera. Transactions of the American Entomological Society. 99:507-546.

Submitted 10 March 2017, Accepted 7 April 2017, Published 1 May 2017

Hosted and published at the University of Illinois, Illinois Natural History Survey, Champaign, Illinois, U.S.A. 